\title{
The Diagnosis and Management of Pediatric Spine Infections
}

\author{
Ehab S. Saleh ${ }^{1}$, Christopher C. Vasileff ${ }^{1}$, Abdullah M. Omari ${ }^{2}$, Jad G. Khalil ${ }^{1}$ \\ 1. Department of Orthopedics, Oakland University William Beaumont School of Medicine, Rochester, USA 2. \\ Department of Orthopedics, William Beaumont Hospital, Taylor, Taylor, USA
}

Corresponding author: Ehab S. Saleh, ehabsaleh@hotmail.com

\begin{abstract}
The management of pediatric spine infections requires a multidisciplinary approach that includes orthopedic surgeons, infectious disease specialists, interventional radiologists, and others.
\end{abstract}

The prevalence of the disease has increased in frequency, virulence, and degree of soft tissue involvement over the past several years; there has also been a resurgence of some types of infections, such as tuberculosis, fungal, and viral pathogens.

The diagnosis can often be reached with a detailed history, physical examination, laboratory tests, and imaging studies. Pathologies mimicking infection require a more invasive approach for diagnosis, including core or open biopsy.

The treatment of discitis, spondylodiscitis, vertebral osteomyelitis, spinal epidural, and intramedullary abscesses in children is at times complex, and although many infections can be treated non-surgically with antibiotic therapy, some more extensive infections require surgical management.

A timely diagnosis is important as it allows the initiation of the appropriate antimicrobial therapy and would decrease the complexity of the subsequent surgical intervention.

Categories: Orthopedics

Keywords: spondylodiscitis, vertebral osteomyelitis, tubercular osteomyelitis, epidural abscess, ct-guided biopsy

\section{Introduction And Background}

Spinal infection in children includes infection of the spinal cord, the nerve roots, meninges, the vertebra, the intervertebral disc, the epidural, intradural, and intrathecal space, and post-operative infections [1]. The incidence of spinal infection in patients less than 20 years old living in developed countries is 0.3 per $100,000[1]$.

Review began 07/06/2021 Review ended 07/24/2021 Published 07/30/2021

\section{๑) Copyright 2021}

Saleh et al. This is an open access article distributed under the terms of the Creative Commons Attribution License CC-BY 4.0., which permits unrestricted use, distribution, and reproduction in any medium, provided the original author and source are credited.
The most common organism causing pyogenic infection of the spine in children is methicillin-sensitive Staphylococcus aureus, with Kingella kingae being especially common in patients between six months and four years of age $[2,3]$.

Erythrocyte sedimentation rate was found to be more sensitive than C-reactive protein in aiding with the diagnosis in one cohort (78\% versus 38\%) [4].

Pediatric spinal infections have a triphasic age distribution; the first is in early infancy, the second is between six months and four years, and the third is in school-aged children [5]. The mean age at presentation is 4.3 years (range is from one to 15 years), and the female to male ratio is 1.3:1 [4].

In the pre-antibiotic era, pediatric spinal infections had a $90 \%$ mortality rate, which has dropped to less than $5 \%$, still a significant number. If the spinal infection is missed or treated late, this can lead to spinal deformities, instabilities, and neurologic complications [5].

Several factors pertaining to the pediatric population can make the diagnosis and treatment more challenging; these include difficulty obtaining a history in non-verbal children, low sensitivity of blood cultures, CT-guided biopsy, non-specific findings of imaging studies in the early stages of the disease, and the lack of consensus on the best treatment approach $[4,5]$.

\section{Review}

\section{Anatomical considerations}

The presence of pure discitis in children has been extensively debated among researchers, but the current 
available evidence indicates that the nucleus pulposus is avascular in both children and adults, and the cartilage endplate and annulus fibrosus receive variable blood supply though life. Pyogenic spondylitis in both age groups is a process that starts in the vertebral endplate [4]. Some similarities and differences of spondylodiscitis in adults and children are summarized in Table $1[2,6]$.

\begin{tabular}{|c|c|c|}
\hline & Pediatric & Adult \\
\hline $\begin{array}{l}\text { The initial seeding } \\
\text { focus of the } \\
\text { infection in the } \\
\text { spine }\end{array}$ & Vertebral endplate & Vertebral endplate \\
\hline $\begin{array}{l}\text { The incidence of } \\
\text { the infection }\end{array}$ & $\begin{array}{l}0.3 \text { per } 100,000 \text { cases per year in persons } \\
\text { less than } 20 \text { years }\end{array}$ & $\begin{array}{l}\text { The overall incidence of spinal infections across all ages per } \\
\text { year is } 2.4 \text { per } 100,000 \text {, This incidence Increases with age to } 6.5 \\
\text { per } 100,000 \text { per year in the } 50-70 \text { years of age group }\end{array}$ \\
\hline $\begin{array}{l}\text { Vascularity of the } \\
\text { disc }\end{array}$ & $\begin{array}{l}\text { Avascular nucleus pulposus, with variable } \\
\text { vascularity of the endplate and the annulus } \\
\text { fibrosus in children and adults through life }\end{array}$ & The same \\
\hline $\begin{array}{l}\text { Delay in } \\
\text { diagnosis }\end{array}$ & Common & Common \\
\hline $\begin{array}{l}\text { Most common } \\
\text { organism }\end{array}$ & Staphylococcus aureus & staphylococcus aureus \\
\hline $\begin{array}{l}\text { Common source } \\
\text { of infection }\end{array}$ & $\begin{array}{l}\text { Subtle bacteremia from an ear, nose, and } \\
\text { throat infection }\end{array}$ & $\begin{array}{l}\text { Obvious bacteremia from intravenous drug use, urinary tract } \\
\text { infection, and other comorbidities }\end{array}$ \\
\hline Treatment & $\begin{array}{l}\text { Mostly intravenous antibiotics with surgery } \\
\text { occasionally required }\end{array}$ & $\begin{array}{l}\text { Mostly intravenous antibiotics with surgery occasionally } \\
\text { required }\end{array}$ \\
\hline Comorbidities & Not common & Common \\
\hline CI-guided biopsy & $\begin{array}{l}\text { Not routinely done, only needed in chronic } \\
\text { cases, atypical cases, and cases not clinically } \\
\text { responding to antibiotics }\end{array}$ & cases, and cases not clinically responding to antibiotics \\
\hline
\end{tabular}

\section{TABLE 1: A comparison between pediatric and adult spondylodiscitis}

A study that retrospectively evaluated 103 children with spinal infection over a 10 -year period found that none presented with only discitis. Spondylodiscitis (infection of the disc and vertebral body) was the primary presentation for toddlers, and vertebral osteomyelitis affected older children and adolescent more frequently. They concluded that pure discitis does not occur, and that a pyogenic infection will start at the metaphyseal region of the vertebra and then spread to adjacent vertebra and the intervening disc space [2].

A unique anatomical feature of the spine, which might lead to multilevel vertebral infection, is the presence of the dural space that is filled with fat and the valveless Batson's venous plexus. Both the venous plexus and the fat may form a route for the dissemination of infection between multiple levels [6].

\section{Pathogenesis}

The usual route of spinal infection in the pediatric population is through a transient minor hematogenous bacteremia [4]. It can also be due to direct inoculation from a diagnostic or surgical procedure, following trauma or direct inoculation from an adjacent genitourinary, gastrointestinal, or oropharyngeal infection $[5,7]$.

Pediatric blunt trauma or hyperextension cervical spine injury has been reported to be a causative factor in delayed cervical spine osteomyelitis, due to a possible small pharyngeal perforation that seeds the bone and eventually develops into osteomyelitis [8].

Spinal infections that happen as a complication of intrathecal baclofen treatment in children with cerebral palsy highly correlate with a gross motor function classification system level five, the presence of gastrostomy tubes, and a history of seizures [9].

Five cases of cervical spine spondylodiscitis have been reported following button battery ingestion in 
children less than five years old, and the battery gets lodged in the esophagus, which leads to local injury, esophageal pressure necrosis, and perforation. The presentation is usually within one to two weeks of the foreign body removal. All reported cases were treated with intravenous antibiotics [10].

Pediatric spine infections can be pyogenic, like vertebral osteomyelitis and spondylodiscitis, or nonpyogenic, like parasitic, fungal, and tuberculous infection of the spine [1]. Methicillin-sensitive and methicillin-resistant Staph are the most common pathogens [1,2]. Other bacterial pathogens include Kingella kingae, group B streptococci, Escherichia coli, Listeria species, Haemophilus influenzae, Neisseria meningitides, and Brucella species [1,2].

Bartonella henselae, the causative bacteria of cat scratch disease, is a rare cause of multifocal osteomyelitis involving the spine and pelvis [11].

Lemierre disease, a rare cause of pediatric spondylodiscitis and epidural abscess, is caused by the anaerobe Fusobacterium necrophorum. These bacteria infect the pharynx initially and then spread to the spine through metastatic abscess formation [12].

Viral pathogens include the herpes virus, poliovirus, and cytomegalovirus [1]. Anogenital Herpes simplex virus (HSV) infection in adolescent patients can cause sacral myeloradiculitis that presents with acute urinary retention, constipation, perineal paresthesia, and erectile dysfunction in the setting of a genital herpes infection [7].

Fungal pathogens include Aspergillus and Candida species [1,13]. Parasitic causes include cysticercosis, schistosomiasis, toxoplasmosis, and echinococcal disease $[1,14]$.

In sickle cell anemia patients, the most common causative organisms are Staphylococcus aureus and salmonella; but vaso-occlusive crisis should always be on the differential diagnosis of a sickle cell child presenting with musculoskeletal pain as there is a low incidence of osteoarticular bacterial infection in this category of children (1.6\%) [15].

\section{Diagnosis}

\section{a. History and Physical Examination}

A triphasic distribution of primary spinal infections exists with $79 \%$ of cases presenting between the ages of six months and four years, $20 \%$ in the juvenile and adolescent age group, and $1 \%$ in children under six months of age [2].

Pediatric spondylodiscitis symptoms depend on the age of the child, with infants presenting with irritability and toddlers presenting with a limp, refusal to sit or walk, and abdominal pain; 75\% will not have fever. Older children might present with back pain and point tenderness [4].

The diagnosis is often delayed (two days to 11.7 months with a mean of 27 days) [2].

\section{b. Laboratory Analysis}

The white blood cell (WBC) count and C-reactive protein (CRP) are mostly normal. The erythrocyte sedimentation rate (ESR) is usually increased to more than $50 \mathrm{~mm} /$ hour in $94 \%$ of the cases [4]. Analysis of serial ESR measurements confirmed this test to be the most accurate for plotting the clinical course of the condition $[1,16,17]$.

Blood cultures in children with pyogenic spine infection are usually negative in the indolent illness presenting with vague back pain and positive in the acute febrile cases, and when they are positive, $S$. aureus is the most common organism isolated $[16,18]$.

\section{c. Imaging}

1. X-ray: This is not a sensitive study to diagnose spondylodiscitis; it can remain normal for two to eight weeks (Figure 1). X-ray findings can include irregularity of the vertebral endplate, disc space narrowing, and lack of definition of the vertebral endplates. In untreated cases, bony sclerosis may begin to appear in 10-12 weeks [19]. 


\section{Cureus}

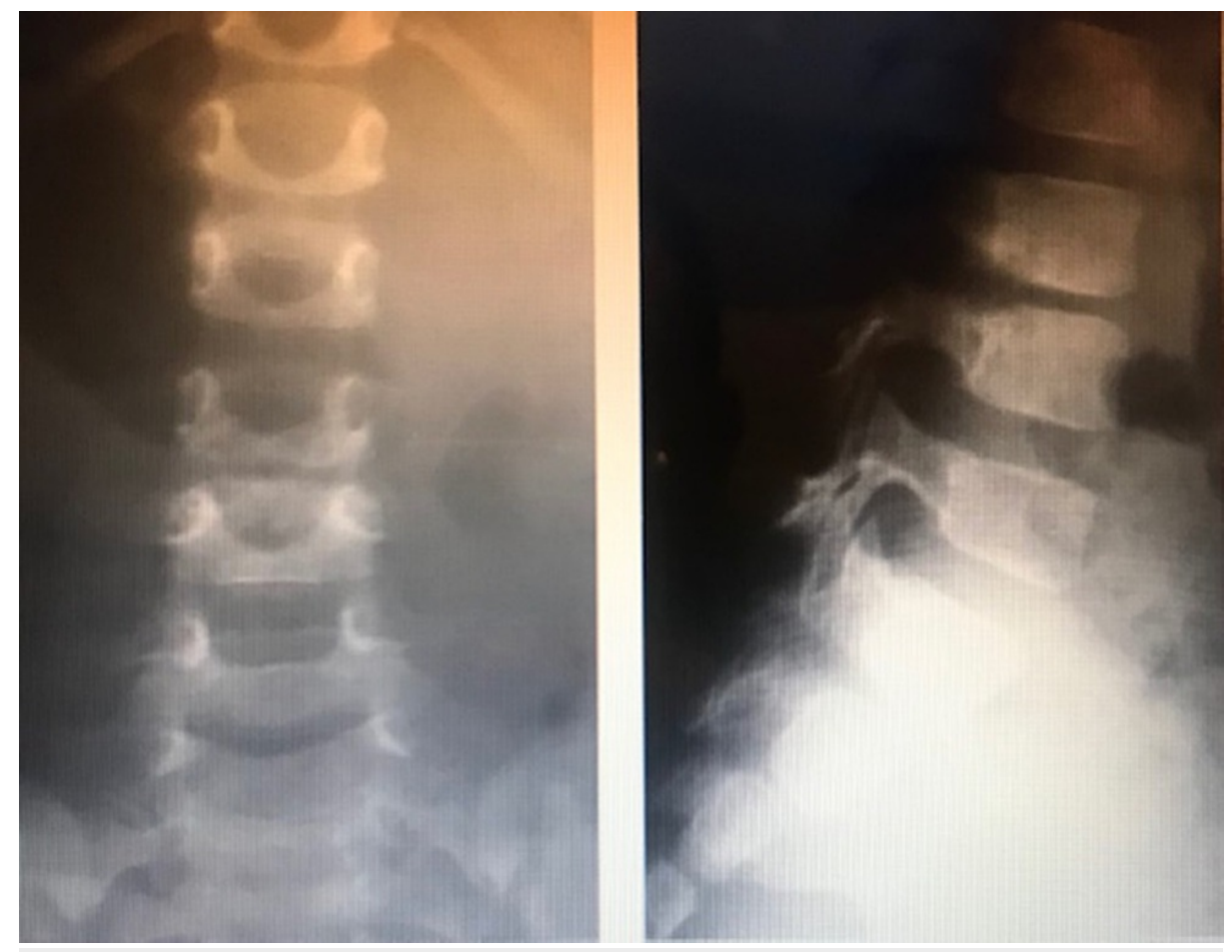

FIGURE 1: Lumbar spine x-ray of a three-year-old child with a two-week history of irritability, showing disk space narrowing of L3-L4 consistent with spondylodiscitis

2. MRI: For the diagnosis of pediatric spine infections, MRI is more sensitive than $\mathrm{x}$-rays or CT scan and more specific than bone scans. Its sensitivity is $96 \%$, and specificity is $93 \%$ for an accuracy of $94 \%$. An infection will show as a region of low $\mathrm{T} 1$ and high $\mathrm{T} 2$ signal intensity, with post-contrast enhancement on fat-suppressed T1-weighted images (Figure 2) [20,21]. 


\section{Cureus}

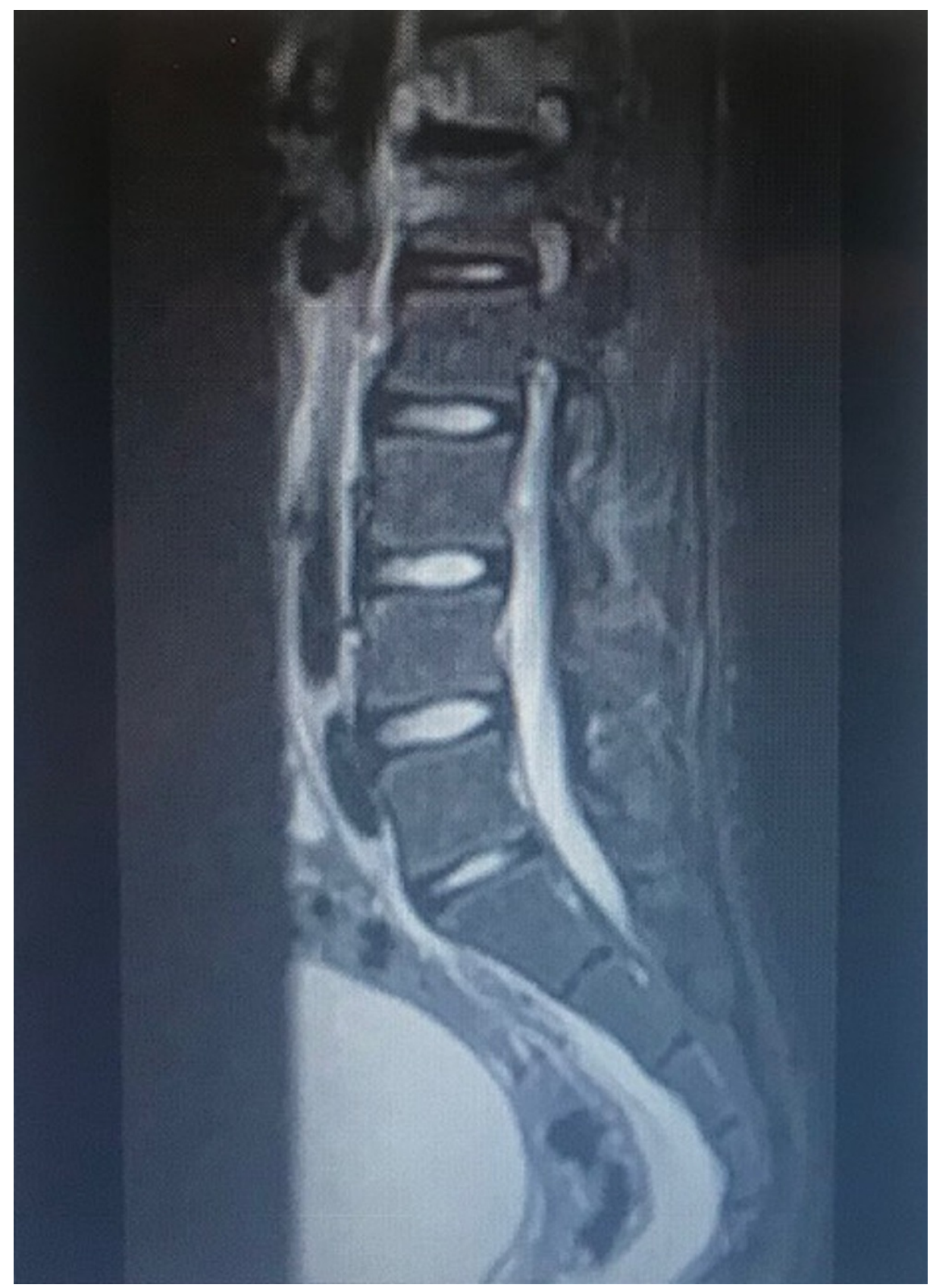

FIGURE 2: MRI of the lumbar spine of a 14-year-old child with spondylodiscitis involving the T12 and L1 vertebras and disc

MRI, Magnetic resonance imaging.

3. 18F-FDG PET/CT: Fluorodeoxyglucose (FDG)-positron emission tomography (PET) CT scan is helpful in diagnosing spine infection when there is retained hardware, where an MRI will be affected by the metal artifact; it can also diagnose other hardware complications not related to infection and other extraspinal sources of infection (Figure 3) [22]. It is superior to Gallium-67 citrate and Technetium-99m bone scan and may be superior to MRI for detection of early or low-grade spondylodiscitis [21]. 


\section{Cureus}

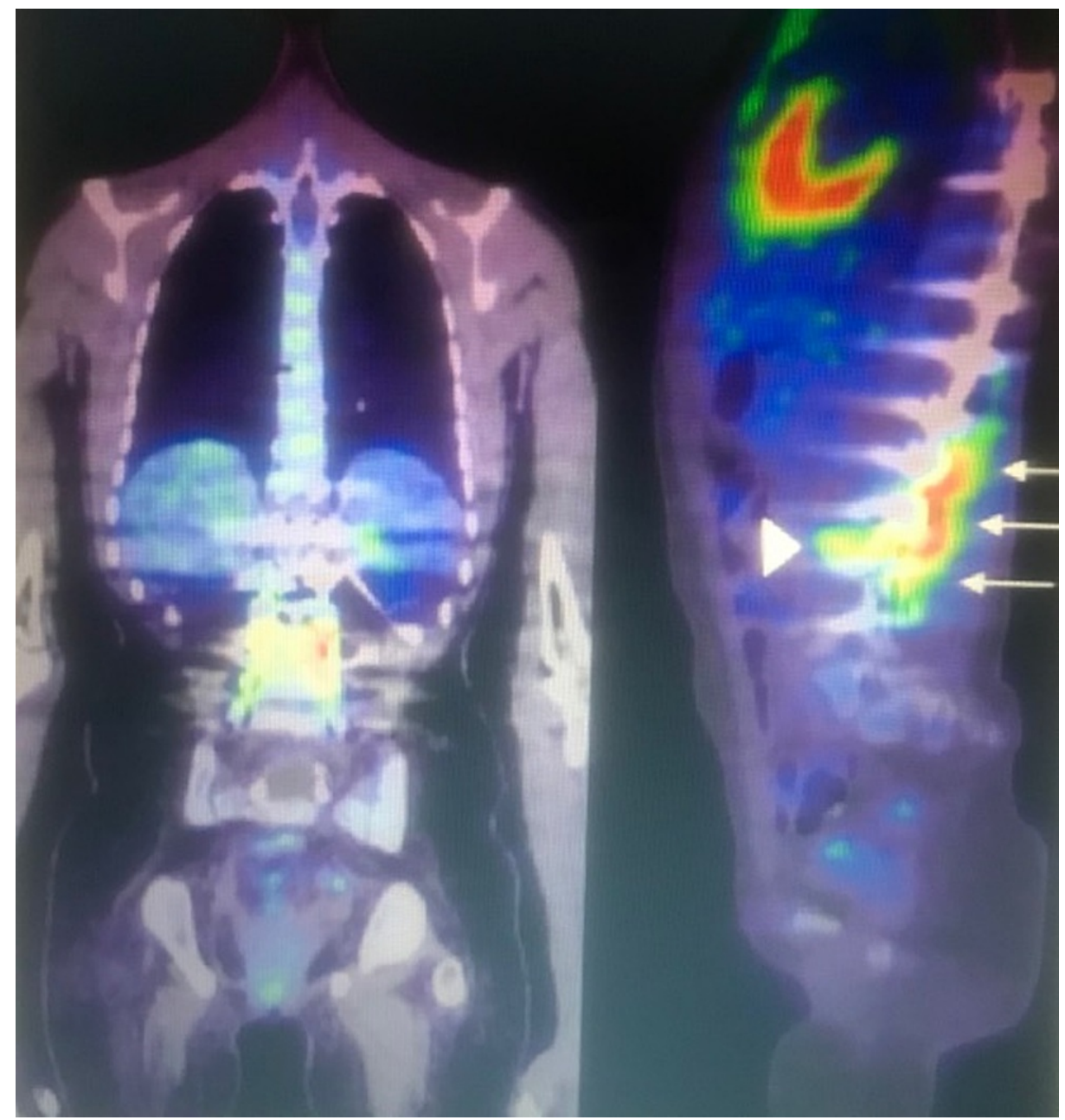

FIGURE 3: Thoracolumbar spinal hardware infection in a 14-year-old child. Coronal and sagittal 18f-FDG PET/CT views of the spinal hardware extending from T6 to L4.

There is increased FDG uptake in the bone and soft tissues immediately adjacent to the hardware from T12 to L4 (arrows). FDG uptake at the bone hardware interface is present at the left L3 interpedicular screw (arrowheads).

FDG, Fluorodeoxyglucose; PET, positron emission tomography; CT, computed tomography.

4. CT-guided biopsy: Spinal biopsy for cultures is not routinely done, and therapy is usually guided by clinical and radiological findings, but in chronic cases, atypical cases, and cases not clinically responding to IV antibiotic, a CT-guided biopsy can yield an organism if blood cultures were negative, which can determine the activity of the disease and guide treatment [18]. The success rate of CT-guided biopsy in finding an organism is between $36 \%$ and $91 \%$ [1], compared to a yield of $76 \%$ for open surgical biopsy [21].

An adult study to evaluate the impact of CT-guided biopsy on patients with suspected spondylodiscitis concluded that an initial CT-guided biopsy will yield a positive culture in about one-third of the cases; $75 \%$ of those initial positive cultures were useful in tailoring the treatment, but $96 \%$ of the study patients without atypical course and with negative blood cultures would have been successfully treated with an empirical course of antibiotic, without needing the CT-guided biopsy result [23].

\section{Clinical Categories}

a. Childhood Spondylodiscitis

Spondylodiscitis is the most common term used to describe primary pyogenic spinal infections that include discitis, spondylodiscitis, and vertebral osteomyelitis. Childhood spondylodiscitis has three clinical forms: the neonatal form (under six months), the infantile form (six months to four years), and a third form affecting children over four years of age including adolescents [2]. 


\section{Cureus}

Spondylodiscitis seems to be the primary pathology in toddlers, whereas vertebral osteomyelitis affects older children and adolescents more $[2,24]$.

b. Tuberculous Infection of the Spine

Tuberculosis of the spine continues to be a problem in many parts of the world, and the spine is involved in $50 \%$ of cases of musculoskeletal tuberculosis, usually presenting with kyphosis, and neurological deficit (Figure 4) [25].

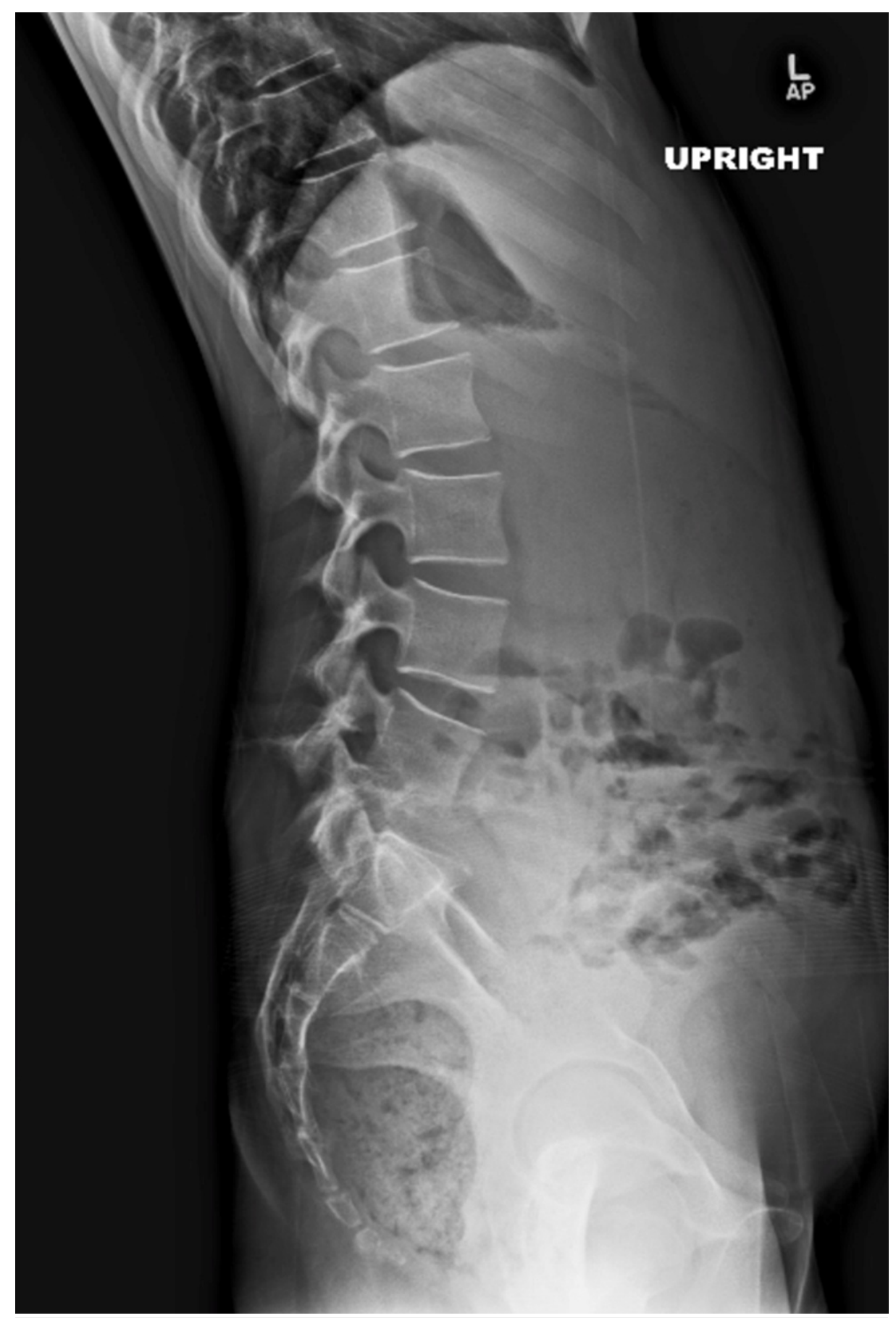

\section{FIGURE 4: A lateral lumbar spine x-ray of a 12-year-old female}

The image shows a lateral lumbar spine x-ray of a 12-year-old female that presented to our institution with a four-month history of back pain, showing loss of the disc space and junctional kyphosis at L4/L5. She was diagnosed with tuberculosis of the lumbar spine. 


\section{Cureus}

cause of pediatric paraplegia in developing countries [26]. But generally, paraplegia is rare in children, compared to adult patients with spinal tuberculosis [1].

Conventional tuberculosis cultures will produce results in four to six weeks, and they have a low yield. A better and faster way of laboratory diagnosis of tuberculosis is with nucleic acid amplification test, which will detect mycobacterial genes after gene amplification via a polymerase chain reaction [26].

On MRI, the presence of multiple vertebral levels involvement with intervening normal levels (skip lesions) is suggestive of tuberculosis over pyogenic spondylodiscitis (Figure 5) [21].

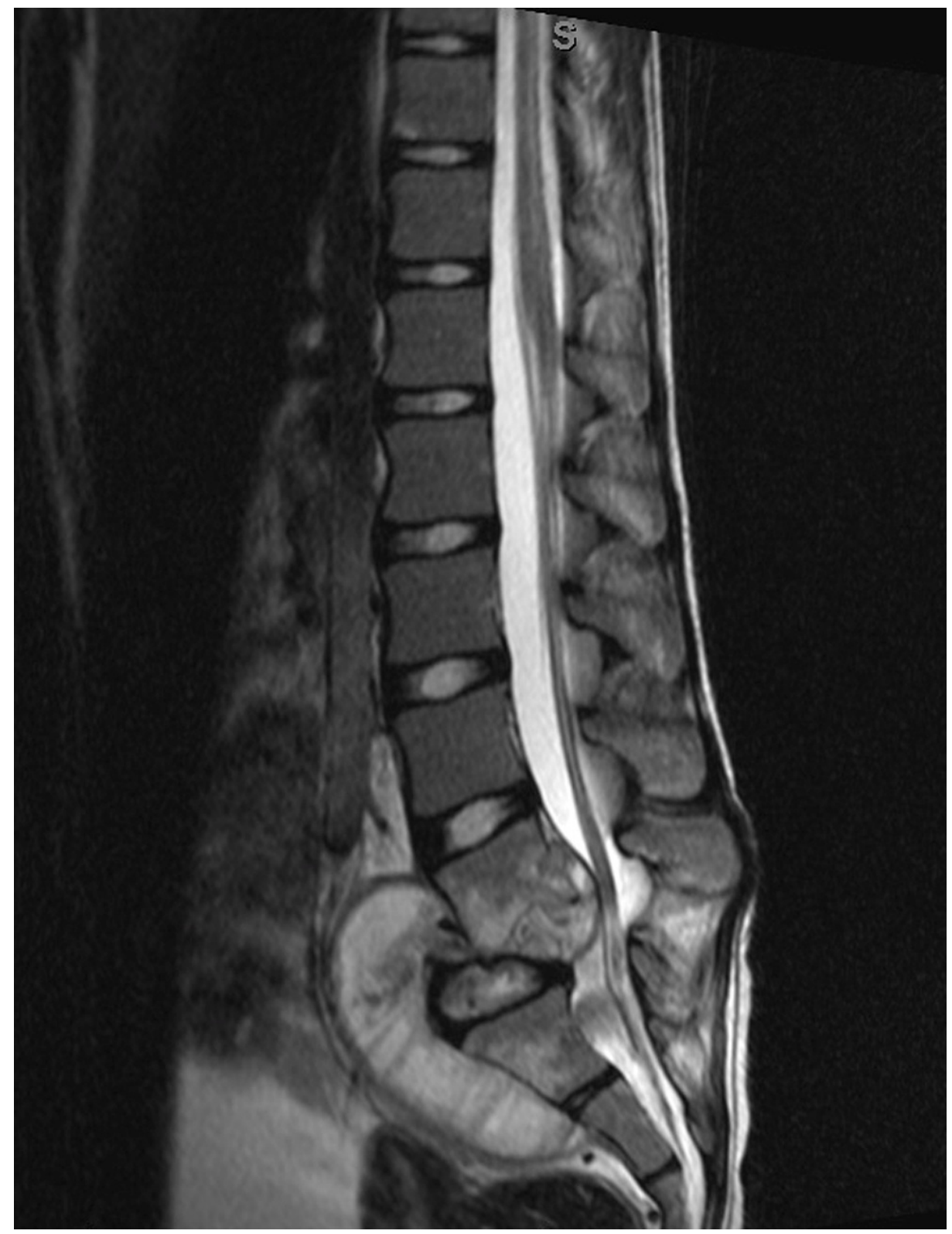

FIGURE 5: Sagittal MRI image of the lumbar spine of the same 12-yearold female with tuberculosis, showing multilevel involvement from L4 to S1 and a large anterior phlegmon

Spinal tuberculosis without structural damage is generally treated with antibiotics and does not usually require surgery if diagnosed and treated early [27].

Indications for surgery include neurological deficits, spinal instability, severe and progressive kyphosis, lack of response to antitubercular agents, and a large paraspinal abscess [25].

Multiple authors reported their good results with surgical treatment using anterior decompression and bone 


\section{Cureus}

grafting, with posterior instrumentation and fusion for cases with neurological deficits not responding to three weeks of antibiotics therapy, and cases with progressive spinal instability and kyphosis (Figure 6) [25$27]$.

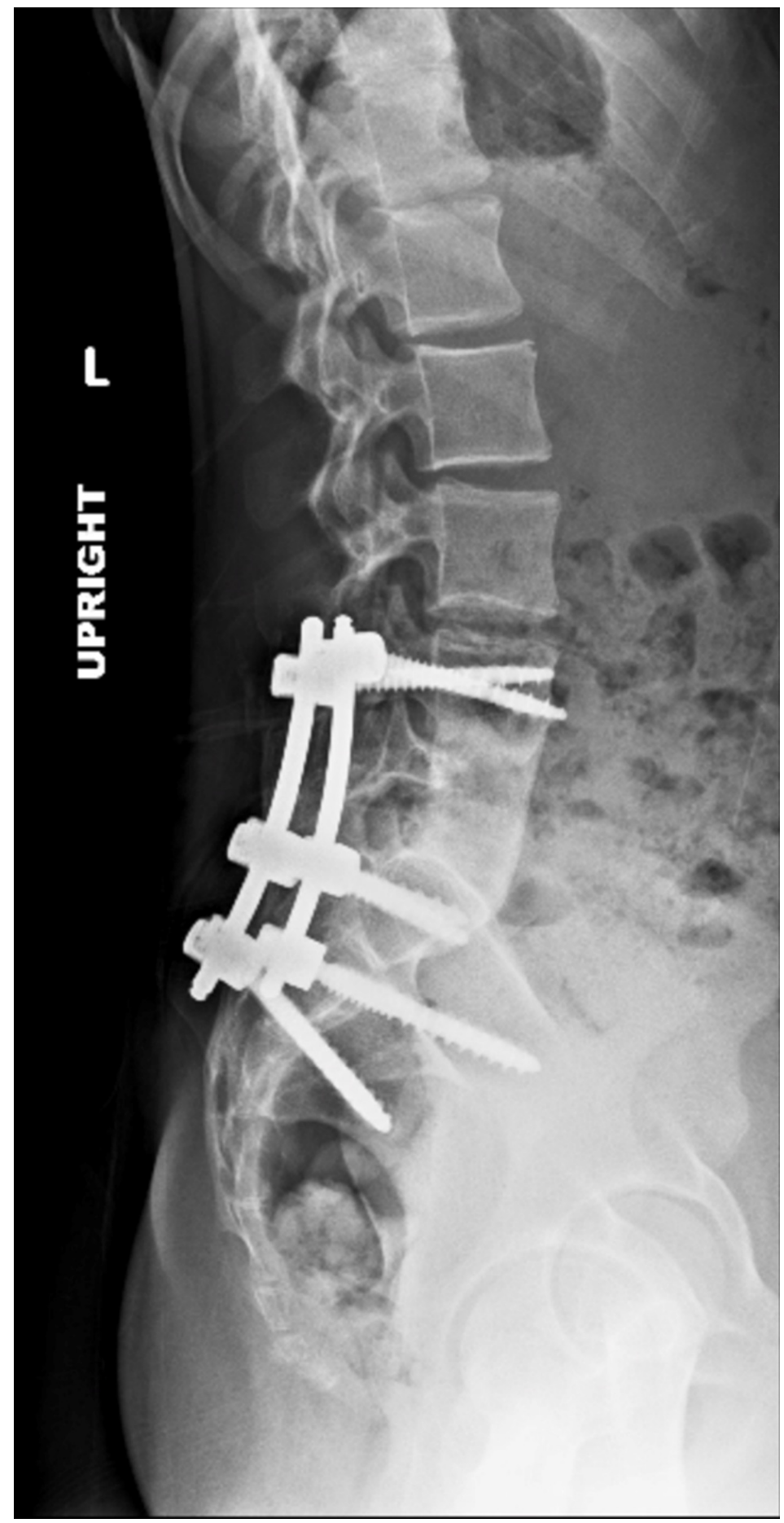

FIGURE 6: Image of the same 12-year-old girl with tuberculosis, after anterior L5 vertebrectomy, lumbar abscess debridement, anterior L4-S1 arthrodesis using tricortical allograft, and posterolateral arthrodesis of L4-S1

c. Cat Scratch Disease

Cat scratch disease is caused by Bartonella henselae, a gram-negative bacillus [28]; 50\% of osteomyelitis caused by cat scratch disease involves the spine. Serology is the main microbiological diagnostic test, and it 
is mostly treated with antibiotics alone, and $20 \%$ of patients might require surgery with an overall good prognosis [29].

A series of five cases of pediatric cervical spine osteomyelitis caused by Bartonella henselae have been reported in the literature in which four of them were treated with antibiotics and surgery, and one was treated with antibiotics alone. The cases that were managed surgically required discectomy and fusion, laminectomy and drainage, anterior open biopsy and drainage, and traction followed by surgical drainage [28].

\section{d. Spinal Epidural Abscess}

Childhood spondylodiscitis can be complicated with soft tissue abscess [2]. Earlier reports on pediatric spinal epidural abscess recommended early surgical drainage as the treatment of choice for all patients [30]. More recent reports did show that pediatric spinal epidural abscesses can be treated successfully with antibiotics alone or in combination with CT-guided drainage, with only a few needing formal surgical drainage $[31,32]$.

Only $8 \%$ of pediatric patients with spinal epidural abscess present with the classic triad of fever, back pain, and neurologic deficit, and 33\%-65\% have no risk factors [32]. Staphylococcus aureus is the most frequently isolated organism [30-32]. As mentioned earlier, Lemierre disease is a rare cause of pediatric spondylodiscitis and epidural abscess formation [12].

\section{e. Spinal Subdural Infection}

Spinal subdural infection is the least common infection in the central nervous system. A total of $53 \%$ of spinal subdural infections in children were associated with spinal dysraphism and other congenital abnormalities of the spine. The most common organism internationally to infect the spinal subdural space in children is Mycobacterium tuberculosis followed by Echinococcus granulosus, Staphylococcus species, and Streptococcus species. The disease is usually treated surgically, although a more expectant approach consisting of antibiotics and observation has also been proposed [33].

\section{f. Intramedullary Spinal Cord Abscess}

This is a rare entity with less than 50 pediatric cases reported since 1830. In children, the most common cause is a congenital dermal sinus with acute cases presenting with fever, pain, and neurological deficit, whereas chronic cases present as an intramedullary tumor. Surgical treatment with multilevel laminectomy, myelotomy, and drainage of the abscess and six to 12 weeks of antibiotics is the treatment of choice, with $60 \%$ good-to-excellent result $[34,35]$.

\section{g. Spine Candida Infection}

In children, the vertebra is the third most common site affected with candida osteomyelitis after the femur and humerus; inflammatory markers are mildly elevated or normal, and most patients will have two or more infected bones [13]. Because of the chronic nature and the multiple bone involvement characteristics of candida musculoskeletal infections, it can be confused with metastatic cancer and chronic multifocal bacterial osteomyelitis [13]. Advanced imaging studies can show skip lesions on MRI-like tuberculosis [21].

About half can be treated with antifungal agents only and the other half with surgical treatment and antifungal agents, given for six to 12 months, and a minority can be treated with only surgery [13].

\section{h. Parasitic Infections}

Four parasitic infections can involve the spine, cysticercosis, schistosomiasis, toxoplasmosis, and echinococcal disease. Spinal neurocysticercosis is rare, present in $1 \%$ to $6 \%$ of patients diagnosed with neurocysticercosis. It most often involves the lower spinal cord; one of its earliest signs is low back pain with radiculopathy.

Toxoplasmosis is the most common opportunistic central nervous system infection affecting patients with AIDS. However, spinal cord involvement is not common. Spinal echinococcal disease can be confused with spinal tuberculosis, especially that Mycobacterium tuberculosis and Echinococcus are present in the same endemic areas. Surgery with laminectomy and decompression is the treatment of choice for spinal echinococcal disease. Needle aspiration carries a significant risk of cystic rupture and dissemination of the parasite and should be avoided [14].

i. Spine Infections in Cerebral Palsy Patients

The infection rate in cerebral palsy patients after scoliosis fusion surgery ranges from $1.1 \%$ to $15.2 \%$. Deep infections are caused by gram-negative organisms, whereas superficial infections are caused equally by 
gram-positive and gram-negative organisms. The most common organisms are Escherichia coli, Pseudomonas aeruginosa, and gram-positive organisms. Risk factors increasing the rate of infection in cerebral palsy patients include a poor nutritional status, a large curve, the presence of baclofen pump, ventriculoperitoneal shunt, gastrostomy tube, gastroesophageal reflux disease, and increased intraoperative blood loss [36].

\title{
Nonoperative management of pediatric spine infection
}

\author{
a. Antibiotic Treatment
}

Treatment for pediatric spinal infection is usually with empiric intravenous antibiotics. The antibiotics choices in cases presenting clinically with pyogenic spondylodiscitis, without suspicion of an atypical organism, are as follows: (1) for neonates and infants under six months, amoxicillin/clavulanate with gentamicin to cover methicillin-sensitive Staph, Streptococcus agalactia, and gram-negative bacteria; (2) for infants between six months and four years, amoxicillin/clavulanate or cefuroxime to cover Kingella kingae; and (3) for children over four years, flucloxacillin or amoxicillin/clavulanate to cover MSSA [2].

Methicillin-resistant $S$. aureus, should also be considered, Hawkins et al. found six out of nine of their cases of spinal epidural abscess to grow methicillin-resistant $S$. aureus, possibly indicating the changing prevalence and invasiveness of community-acquired MRSA [31].

Spinal epidural abscesses have been traditionally treated with surgical decompression and drainage in combination with antibiotics [31], although recent literature suggests that surgery can be avoided in favor of antibiotic therapy, in combination with other nonoperative treatment modalities like minimally invasive drainage techniques [31,32].

For patients with spinal tuberculosis, treatment includes isoniazid (5-10 mg/kg), rifampicin (10-20 mg/kg), ethambutol in children older than six years $(15 \mathrm{mg} / \mathrm{kg})$, and pyrazinamide $(25 \mathrm{mg} / \mathrm{kg}$ ) for one year [37].

For patients with the rare epidural abscess complicating Lemierre disease, the causative organism Fusobacterium necrophorum will need treatment with IV meropenem for six weeks in addition to surgery [12].

Patients with diagnosed HSV sacral myeloradiculitis have been reported to achieve complete resolution of symptoms after three weeks of IV acyclovir [7].

\section{b. Interventional Treatment}

CT-guided biopsy is reserved for cases resistant to antimicrobial therapy or when there are concerns regarding other pathology mimicking infection, due to its low rate of positive cultures [4]. In instances that a CT-guided biopsy is indicated, positive cultures have been shown to reliably provide helpful information in guiding antibiotic treatment [23]. Cases of spinal infection with uncommon causes may require lumbar puncture in order to perform cerebrospinal fluid analysis [7].

\section{Operative management of pediatric spine infections}

Although rare, some cases of pediatric spinal infections do require surgical intervention, and those cases are crucial to identify. Generally, surgery is reserved for patients displaying progressive neurologic deficit or progressive instability [38]. The indications for decompression and atlantoaxial fusion for cervical spine tuberculosis in children are neurologic deficit, atlantodental interval greater than $5 \mathrm{~mm}$ on flexion/extension views, and progressive deformity [37].

In pediatric spinal tuberculosis throughout the spinal column for patients with active tuberculosis, posterior instrumented stabilization combined with anterior radical debridement should be reserved only for advanced tuberculosis with instability, rapid progress of kyphosis, and unacceptable pre-existing kyphosis [27].

A novel surgical treatment of a three-month-old infant with vertebral osteomyelitis displaying neurological decline from progressive instability of the occiput, C1, and C2 levels after failed bracing was described [38]. The patient had reduction and occiput to $\mathrm{C} 2$ posterior fusion and segmental instrumentation, with nonsegmental instrumentation extending in the soft tissues from $\mathrm{C} 2$ to $\mathrm{T} 4$ with the intent of providing stabilization without fusion and permitting further growth. This technique was used in order to overcome the difficulty of providing secure immobilization of the craniocervical junction while not creating an inadvertent fusion of the sub-axial cervical spine.

\section{Complications of pediatric spine infections}

Children with spinal infections generally have good functional outcomes after completing treatment, in part due to their remodeling potential [37]. Poor outcomes, however, do unfortunately occur. Many of these complications are seen in patients that undergo operative management, but some are seen in patients 
managed conservatively as well. Patients with a history of spondylodiscitis can have persistent intermittent back pain usually with little to no impact on daily activities [4]. Some patients with spondylodiscitis of the cervical spine, however, can develop auto fusion or deformity of adjacent vertebrae leading to a significant decrease in neck range of motion and subsequent debility [24]. Patients with spondylodiscitis of the thoracic or lumbar spine are at the risk of developing scoliosis or kyphosis, sometimes requiring surgical correction $[7,24]$. Glotzbecker et al. noted in their case report of neonatal axial spine osteomyelitis that the patients continued to have mild global developmental delay two years following successful hardware removal [38]. Historical literature has shown that non-surgical management of spinal infections in children frequently leads to paraplegia [27] and was routinely fatal [30], but the adoption of modern practices has led to massive improvements in both morbidity and mortality.

\section{Differential diagnosis of pediatric spine infection}

Conditions to be considered in the differential diagnosis in a child presenting with back pain and a lytic lesion of the spine include eosinophilic granulomas, leukemia, lymphoma, fibrous dysplasia, and Ewing's sarcoma.

\section{Conclusions}

Albeit a rare occurrence in clinical practice, having a low threshold of suspicion is needed when faced with possible pediatric spine infection as early recognition and prompt initiation of appropriate treatment are paramount in achieving a satisfactory resolution of the disease. Invasive diagnostic procedures should be considered in atypical and chronic cases as well as in cases not responding to IV antibiotics. Other differential diagnoses that are common which the physicians should be aware and exclude efficiently includes eosinophilic granulomas, leukemia, lymphoma, fibrous dysplasia, or Ewing's sarcoma.

\section{Additional Information}

\section{Disclosures}

Conflicts of interest: In compliance with the ICMJE uniform disclosure form, all authors declare the following: Payment/services info: All authors have declared that no financial support was received from any organization for the submitted work. Financial relationships: All authors have declared that they have no financial relationships at present or within the previous three years with any organizations that might have an interest in the submitted work. Other relationships: All authors have declared that there are no other relationships or activities that could appear to have influenced the submitted work.

\section{References}

1. Tyagi R: Spinal infections in children: a review . J Orthop. 2016, 13:254-8. 10.1016/j.jor.2016.06.005

2. Dayer R, Alzahrani MM, Saran N, et al.: Spinal infections in children: a multicentre retrospective study . Bone Joint J. 2018, 100-B:542-8. 10.1302/0301-620X.100B4.BJJ-2017-1080.R1

3. Yagupsky P: Kingella kingae: carriage, transmission, and disease. Clin Microbiol Rev. 2015, 28:54-79. 10.1128/CMR.00028-14

4. Afshari FT, Rodrigues D, Bhat M, Solanki GA, Walsh AR, Lo WB: Paediatric spondylodiscitis: a 10-year single institution experience in management and clinical outcomes. Childs Nerv Syst. 2020, 36:1049-1054. 10.1007/s00381-019-04470-z

5. Principi N, Esposito S: Infectious discitis and spondylodiscitis in children. Int J Mol Sci. 2016, 17:539. 10.3390/ijms 17040539

6. Song KS, Ogden JA, Ganey T, Guidera KJ: Contiguous discitis and osteomyelitis in children . J Pediatr Orthop. 1997, 17:470-7.

7. Whalen AM, Mateo CM, Growdon AS, Miller AF: Sacral myeloradiculitis: an uncommon complication of genital Herpes infection. Pediatrics. 2019, 144: 10.1542/peds.2018-2631

8. Palmer V, Cohen RB, Braffman B, Brockmeyer DL, Spader HS: Delayed osteomyelitis resulting from an extension injury of the cervical spine: case report. J Neurosurg Pediatr. 2017, 20:388-92. 10.3171/2017.5.PEDS1777

9. Bayhan IA, Sees JP, Nishnianidze T, Rogers KJ, Miller F: Infection as a complication of intrathecal baclofen treatment in children with cerebral palsy. J Pediatr Orthop. 2016, 36:305-9. 10.1097/BPO.0000000000000443

10. Young A, Tekes A, Huisman TA, Bosemani T: Spondylodiscitis associated with button battery ingestion: prompt evaluation with MRI. Neuroradiol J. 2015, 28:504-7. 10.1177/1971400915611142

11. Erdem G, Watson JR, Hunt WG, et al.: Clinical and radiologic manifestations of bone infection in children with cat scratch disease. J Pediatr. 2018, 201:274-280.e12. 10.1016/j.jpeds.2018.05.033

12. Sinatra PM, Alander DH: Lemierre disease: a case with multilevel epidural abscess and aggressive neurological weakness: case report and literature review. J Pediatr Orthop. 2017, 37:e58-61. 10.1097/BPO.0000000000000652

13. Gamaletsou MN, Kontoyiannis DP, Sipsas NV, et al.: Candida osteomyelitis: analysis of 207 pediatric and adult cases (1970-2011). Clin Infect Dis. 2012, 55:1338-51. 10.1093/cid/cis660

14. Majmundar N, Patel PD, Dodson V, Tran A, Goldstein I, Assina R: Parasitic infections of the spine: case series and review of the literature. Neurosurg Focus. 2019, 46:E12. 10.3171/2018.10.FOCUS18472

15. Dalton GP, Drummond DS, Davidson RS, Robertson WW Jr: Bone infarction versus infection in sickle cell disease in children. J Pediatr Orthop. 1996, 16:540-4. 10.1097/00004694-199607000-00024

16. Chandrasenan J, Klezl Z, Bommireddy R, Calthorpe D: Spondylodiscitis in children: a retrospective series. J 
Bone Joint Surg Br. 2011, 93:1122-5. 10.1302/0301-620X.93B8.25588

17. Spencer SJ, Wilson NI: Childhood discitis in a regional children's hospital . J Pediatr Orthop B. 2012, 21:2648. 10.1097/BPB.0b013e32834d3e94

18. Hoffer FA, Strand RD, Gebhardt MC: Percutaneous biopsy of pyogenic infection of the spine in children . J Pediatr Orthop. 1988, 8:442-4. 10.1097/01241398-198807000-00011

19. Fucs PM, Meves R, Yamada HH: Spinal infections in children: a review . Int Orthop. 2012, 36:387-95. 10.1007/s00264-011-1388-2

20. Khanna AJ, Wasserman BA, Sponseller PD: Magnetic resonance imaging of the pediatric spine. J Am Acad Orthop Surg. 2003, 11:248-59. 10.5435/00124635-200307000-00004

21. Talbott JF, Shah VN, Uzelac A, Narvid J, Dumont RA, Chin CT, Wilson DM: Imaging-based approach to extradural infections of the spine. Semin Ultrasound CT MR. 2018, 39:570-86. 10.1053/j.sult.2018.09.003

22. Bagrosky BM, Hayes KL, Koo PJ, Fenton LZ: 18F-FDG PET/CT evaluation of children and young adults with suspected spinal fusion hardware infection. Pediatr Radiol. 2013, 43:991-1000. 10.1007/s00247-013-2654-9

23. Kasalak Ö, Wouthuyzen-Bakker M, Adams HJ, Overbosch J, Dierckx RA, Jutte PC, Kwee TC: CT-guided biopsy in suspected spondylodiscitis: microbiological yield, impact on antimicrobial treatment, and relationship with outcome. Skeletal Radiol. 2018, 47:1383-91. 10.1007/s00256-018-2944-2

24. Garron E, Viehweger E, Launay F, Guillaume JM, Jouve JL, Bollini G: Nontuberculous spondylodiscitis in children. J Pediatr Orthop. 2002, 22:321-8.

25. Huang QS, Zheng C, Hu Y, Yin X, Xu H, Zhang G, Wang Q: One-stage surgical management for children with spinal tuberculosis by anterior decompression and posterior instrumentation. Int Orthop. 2009, 33:1385-90. 10.1007/s00264-009-0758-5

26. Chatterjee S, Banta A: The spectrum of tuberculosis of the spine in pediatric age group: a review . Childs Nerv Syst. 2018, 34:1937-45. 10.1007/s00381-018-3891-x

27. Moon MS, Kim SS, Lee BJ, Moon JL: Spinal tuberculosis in children: retrospective analysis of 124 patients . Indian J Orthop. 2012, 46:150-8. 10.4103/0019-5413.93676

28. Akbari SH, Averill CE, Roland JL, Orscheln R, Strahle J: Bartonella henselae infection presenting as cervical spine osteomyelitis: case report. J Neurosurg Pediatr. 2018, 22:189-94. 10.3171/2018.2.PEDS17600

29. Donà D, Nai Fovino L, Mozzo E, et al.: Osteomyelitis in cat-scratch disease: a never-ending dilemma-a case report and literature review. Case Rep Pediatr. 2018, 2018:1679306. 10.1155/2018/1679306

30. Enberg RN, Kaplan RJ: Spinal epidural abscess in children. Early diagnosis and immediate surgical drainage is essential to forestall paralysis. Clin Pediatr (Phila). 1974, 13:247-8 passim. 10.1177/000992287401300309

31. Hawkins M, Bolton M: Pediatric spinal epidural abscess: a 9-year institutional review and review of the literature. Pediatrics. 2013, 132:e1680-5. 10.1542/peds.2012-3805

32. Sugawara R, Kikkawa I, Watanabe H, Hiyama S, Kikuchi Y, Takeshita K: Pediatric spinal epidural abscess: a case report of a 12-year-old girl without risk factors. J Am Acad Orthop Surg Glob Res Rev. 2019, 3:e066. 10.5435/JAAOSGlobal-D-18-00066

33. Sandler AL, Thompson D, Goodrich JT, et al.: Infections of the spinal subdural space in children: a series of 11 contemporary cases and review of all published reports. A multinational collaborative effort. Childs Nerv Syst. 2013, 29:105-17. 10.1007/s00381-012-1916-4

34. Prasad GL: Intramedullary spinal cord abscess-a rare entity in pediatric age group . Childs Nerv Syst. 2016, 32:2285-6. 10.1007/s00381-016-3251-7

35. Prasad GL, Hegde A, Divya S: Spinal intramedullary abscess secondary to dermal sinus in children . Eur J Pediatr Surg. 2019, 29:229-38. 10.1055/s-0038-1655736

36. Sebaaly A, El Rachkidi R, Yaacoub JJ, Saliba E, Ghanem I: Management of spinal infections in children with cerebral palsy. Orthop Traumatol Surg Res. 2016, 102:801-5. 10.1016/j.otsr.2016.04.015

37. Govender S, Ramnarain A, Danaviah S: Cervical spine tuberculosis in children . Clin Orthop Relat Res. 2007, 460:78-85. 10.1097/BLO.0b013e31806a915f

38. Glotzbecker MP, Wasser AM, Troy MJ, Proctor M, Emans JB: Neonatal C1 TO C2 osteomyelitis leading to instability and neurological decline: novel treatment with occiput-C1-C2 fusion and occiput to thorax growing rods. A case report. J Pediatr Orthop. 2015, 35:379-84. 10.1097/BPO.0000000000000309 